\title{
THE ESO SLICE PROJECT (ESP) REDSHIFT SURVEY
}

\author{
G. VETTOLANI ${ }^{1}$, E. ZUCCA ${ }^{2,1}$, A. CAPPI $^{2}$, R. MERIGHI ${ }^{2}$, \\ M. MIGNOLI ${ }^{2}$, G. STIRPE ${ }^{2}$, G. ZAMORANI ${ }^{2,1}$, \\ H. MacGILLIVRAY ${ }^{3}$, C. COLLINS ${ }^{4}$, C. BALKOWSKI ${ }^{5}$, \\ V. CAYATTE ${ }^{5}$, S. MAUROGORDATO ${ }^{5}$, D. PROUST $^{5}$, \\ G. CHINCARINI ${ }^{6}$, L. GUZZO $^{6}$, D. MACCAGNI ${ }^{7}$, \\ R. SCARAMELLA ${ }^{8}$, A. BLANCHARD ${ }^{9}$ AND M. RAMELLA ${ }^{10}$ \\ 1 Istituto di Radioastronomia del CNR, Italy \\ 2 Osservatorio Astronomico di Bologna, Italy \\ 3 Royal Observatory Edinburgh, United Kingdom \\ 4 Liverpool John Moores University, United Kingdom \\ 5 DAEC, Observatoire de Paris-Meudon, France \\ 6 Osservatorio Astronomico di Brera-Merate, Italy \\ 7 Istituto di Fisica Cosmica e Tecnologie Relative, Italy \\ 8 Osservatorio Astronomico di Roma, Italy \\ 9 Universitè Louis Pasteur, France \\ 10 Osservatorio Astronomico di Trieste, Italy
}

\section{The ESO Slice Project Redshift Survey}

The ESO Slice Project (ESP) is a galaxy redshift survey we have recently completed as an ESO Key-Project over about 30 square degrees, in a region near the South Galactic Pole (Vettolani et al., submitted to A\&A). The survey is nearly complete to the limiting magnitude $b_{J}=19.4$ and consists of more than three thousands galaxies with reliable redshift determination.

The ESP survey is intermediate between shallow, wide angle samples and very deep, monodimensional pencil beams: spanning a volume of $\sim 10^{5}$ $h^{-3} \mathrm{Mpc}^{3}$ at the sensitivity peak $(z \sim 0.1)$ it can provide an accurate determination of the "local" luminosity function and the mean galaxy density (Zucca et al., submitted to A\&A). Moreover, it can allow clustering analyses not biased anymore by nearby structures. Finally, this uniform set of spectra will allow us interesting studies about the K-correction and the galaxy evolutionary properties, based on a large homogeneous sample.

Here we report some results about the luminosity function. 


\section{The Luminosity Function}

We find that, although a Schechter function (with $\alpha=-1.22, M_{b_{J}}^{*}=$ $-19.61+5 \log h$ and $\phi^{*}=0.020 h^{3} \mathrm{Mpc}^{-3}$ ) is an acceptable representation of the luminosity function over the entire range of magnitudes $\left(M_{b_{J}} \leq\right.$ $-12.4+5 \log h)$, our data strongly suggest a steepening of the luminosity function for $M_{b_{J}} \geq-17+5 \log h$. Such a steepening, well fitted by a power law with slope $\beta \sim 1.6$, is in agreement with what has been recently found by similar analyses for both field galaxies (Marzke et al. 1994) and galaxies in clusters (e.g., Driver \& Phillipps 1996).

This steepening at the faint end of the luminosity function is almost completely due to galaxies with emission lines: in fact dividing galaxies into two samples, i.e., galaxies with and without emission lines, we find significant differences in their luminosity functions. In particular, galaxies with emission lines (which are $\sim 50 \%$ of the total) show a steeper slope and a fainter $M^{*}$.

The normalization and the $\alpha$ and $M^{*}$ parameters of our luminosity function are in excellent agreement with those of the AUTOFIB redshift survey (Ellis et al. 1996). Viceversa, our normalization is a factor $\sim 2$ higher than that found for both the APM (Loveday et al. 1992) and the Las Campanas (Lin et al. 1996) redshift surveys. Also the faint end slope of our luminosity function is significantly steeper than that found in these two surveys.

The galaxy number density for $M_{b_{J}} \leq-16+5 \log h$ is well determined $\left(\bar{n}=0.08 h^{3} \mathrm{Mpc}^{-3}\right)$. Its estimate for $M_{b_{J}} \leq-12.4+5 \log h$ is more uncertain, ranging from $\bar{n}=0.28 h^{3} \mathrm{Mpc}^{-3}$, in the case of a fit with a single Schechter function, to $\bar{n}=0.54 h^{3} \mathrm{Mpc}^{-3}$, in the case of Schechter function and power law fit. The corresponding luminosity densities in these three cases are $\rho_{L U M}=(2.03,2.23,2.31) \times 10^{8} h \mathrm{~L}_{\odot} \mathrm{Mpc}^{-3}$, respectively.

\section{References}

Driver, S.P., Phillipps, S., 1996, Astrophys.J. in press

Ellis, R.S., Colless, M., Broadhurst, T., Heyl, J., Glazebrook, K., 1996, Mon. Not. R. astron. Soc. 280,235

Lin, H., Kirshner, R.P., Shectman, S.A., et al. 1996, Astrophys.J. 464, 60

Loveday, J., Peterson, B.A., Efstathiou, G., Maddox, S.J., 1992, Astrophys.J. 390, 338

Marzke, R.O., Huchra, J.P., Geller, M.J., 1994, Astrophys.J. 428, 43 